\title{
Tratamento conservativo e cirúrgico em 22 ruminantes com fraturas em membros ${ }^{1}$
}

\author{
Antônio Carlos Lopes Câmara²*, Eraldo Barbosa Calado², João Marcelo A. de Paula \\ Antunes $^{2}$, Camila Marinho de M. Oliveira ${ }^{2}$, José Augusto Bastos Afonso ${ }^{3}$ \\ e Nivaldo de Azevêdo Costa ${ }^{3}$
}

\begin{abstract}
Câmara A.C.L., Calado E.B., Antunes J.M.A.P., Oliveira C.M.M., Afonso J.A.B. \& Costa N.A. 2014. [Conservative and surgical treatment in 22 ruminants with limb fractures.] Tratamento conservativo e cirúrgico em 22 ruminantes com fraturas em membros. Pesquisa Veterinária Brasileira 34(11):1045-1050. Hospital Veterinário, Universidade Federal Rural do Semi-Árido, BR-110 Km 47, Presidente Costa e Silva, Mossoró, RN 59625-900, Brazil. E-mail: aclcamara@yahoo.com.br

The aim of the present study was to report the main locations of limb fractures in 22 ruminants and to determine the efficiency of the treatment choices. Eight goats, eight sheep and six cattle were included. In cases of distal fractures, the conservative treatment with immobilization was the method of choice. In cases of proximal, exposed metatarsal or metacarpal fractures, the ruminants were treated surgically. The higher frequency of fractures involved the metacarpal or metatarsal (54.5\%) followed by fractures of the tibia (22.7\%), femur (9\%) and isolated cases of medial phalanx, humerus, radius and ulna fractures ( $4.5 \%$ each). Total recovery rate reached 95.4\%. Immobilization with plaster associated with Thomas splint, or just with wooden splints in young animals, was efficient in reducing fractures of metacarpal, metatarsal, tibia, humerus, and radio in the treated ruminants. In the case of medial phalanx fracture, immobilization using only plaster was sufficient to provide adequate bone repair. The internal or external skeletal fixation should be considered an option in the treatment of metacarpal, metatarsal, tibial and femoral fractures in ruminants, especially in low body weight animals.
\end{abstract}

INDEX TERMS: Fractures, external skeletal fixator, orthopedics, osteosynthesis, Thomas split, ruminants.

RESUMO.- 0 objetivo do presente trabalho foi relatar os principais locais de fraturas em membros de 22 ruminantes e determinar a eficiência dos tratamentos utilizados. Foram incluídos no estudo oito caprinos, oito ovinos e seis bovinos Em casos de fraturas distais, o tratamento conservativo com imobilização foi o método de eleição. Em casos de fraturas proximais e fraturas expostas de metatarso ou metacarpo, os ruminantes foram tratados cirurgicamente. Observou-se maior frequência de fraturas envolvendo o metacarpo ou metatarso $(54,5 \%)$, seguido por fraturas de tíbia (22,7\%), fêmur (9\%) e casos isolados de fraturas de falange medial, úmero, rádio e ulna $(4,5 \%$ cada). 0 ín-

\footnotetext{
${ }^{1}$ Recebido em 20 de junho de 2014.

Aceito para publicação em 4 de julho de 2014.

${ }^{2}$ Hospital Veterinário, Universidade Federal Rural do Semi-Árido, BR$110 \mathrm{Km} 47$, Mossoró, RN 59625-900, Brasil. *Autor para correspondência: aclcamara@yahoo.com.br

${ }^{3}$ Clínica de Bovinos, Campus Garanhuns, Universidade Federal Rural de Pernambuco, Cx. Postal 152, Garanhuns, PE 55292-901, Brasil.
}

dice de recuperação total alcançou 95,4\%. A imobilização com gesso associada à tala de Thomas, ou apenas com talas de madeira em animais jovens, foi eficiente na redução de fraturas de metacarpo, metatarso, tíbia, úmero e rádio nos ruminantes tratados. Enquanto no caso de fratura de falange medial, a imobilização com uso apenas de gesso foi suficiente para proporcionar reparação óssea adequada. A fixação esquelética externa ou interna deve ser considerada uma opção no tratamento de fraturas metacárpicas, metatársicas, tibiais e femorais em ruminantes, principalmente quando o animal for de baixo peso corporal.

TERMOS DE INDEXAÇÃO: Fraturas, fixador esquelético externo, ortopedia, osteossíntese, tala de Thomas, ruminantes.

\section{INTRODUÇÃO}

As fraturas em ruminantes ocorrem com relativa frequência, traduzindo em perdas econômicas significativas para a cadeia produtiva (Spadeto Júnior et al. 2010). As fraturas em membros são mais comumente encontradas em 
animais jovens, associada, na maioria das vezes, a traumas durante distocia, manuseio ou pisoteio da mãe (Mulon 2013). As fraturas mais usualmente observadas incluem fraturas do metacarpo e metatarso com aproximadamente $50 \%$ dos casos, seguidos por fraturas de tíbia (12\%), rádio e ulna (7\%), e úmero ( $<5 \%$ ) (Rielly et al. 2005, Anderson \& St. Jean 2008). Enquanto, fraturas de fêmur, falanges e do esqueleto axial (mandíbula, vértebras, costelas e pélvis) ocorrem ainda em menor frequência (Steiner \& Anderson 2009).

A decisão de tratamento da fratura é feita após considerar o valor econômico ou genético do animal, a localização, o tipo de fratura, o custo deste tratamento e o prognóstico (Anderson \& St. Jean 2008, Mulon 2013). Entretanto, na medicina veterinária de animais de produção, a eutanásia tem sido a opção mais frequentemente utilizada, principalmente em casos de fraturas de ossos longos (Vechiato et al. 2009). Opções de tratamento variam entre confinamento em baia, coaptação externa, fixação externa e redução aberta com fixação interna; dependendo do tipo de fratura e o osso envolvido (Martens et al. 1998, Gangl et al. 2006, Mulon 2013).

Apesar da existência na literatura nacional de referências sobre o tratamento de casos isolados de fraturas espontâneas (Martins et al. 2001, Nóbrega et al. 2008, Vechiato et al. 2009) ou experimentalmente induzidas (Spadeto Júnior et al. 2010), trabalhos com relação à caracterização das fraturas em ruminantes são escassos. Além disso, a casuística no Hospital Veterinário da Universidade Federal Rural do Semi-Árido (HoVet-UFERSA) confirma a importância desta afecção do sistema locomotor, já que do total de 353 ruminantes atendidos no período de junho de 2009 a dezembro de 2013, 22 casos (6,23\%) foram submetidos a procedimentos ortopédicos. Deste modo, o presente trabalho objetiva relatar os principais locais de fraturas em membros de 22 ruminantes e determinar a eficiência dos tratamentos utilizados.

\section{MATERIAL E MÉTODOS}

As fichas clínicas de ruminantes diagnosticados com fraturas em membros e submetidos a procedimentos ortopédicos foram avaliadas. Os casos foram atendidos no HOVET-UFERSA, em Mossoró/RN, entre junho de 2009 e dezembro de 2013. As informações resgatadas incluíram os dados epidemiológicos, achados clínicos e radiográficos; tratamento instituído e desfecho. Foram incluídos no estudo oito caprinos, oito ovinos e seis bovinos, totalizando 22 ruminantes. Para confirmação da eficiência do tratamento, os ruminantes foram novamente trazidos ao HOVET-UFERSA para retirada da imobilização, entretanto nos casos em que os proprietários não retornaram (8 casos), estes foram contatados por telefone posteriormente para averiguação do retorno à atividade funcional do ruminante.

\section{Imobilização com gesso e/ou tala}

Em casos de fraturas distais (abaixo da articulação carpo-radial ou tarso-tibial), o tratamento conservativo com imobilização foi o método de eleição. Para isso, os animais foram tranquilizados com cloridrato de xilazina (Xilazin - Syntec do Brasil Ltda, Cotia, SP) $\left(0,1 \mathrm{mg}_{\mathrm{kg}} \mathrm{kg}^{-1}\right)$ e mantidos em decúbito lateral contrário ao lado do membro acometido. Em seguida, removeu-se qualquer resíduo orgânico deste membro com posterior alinhamento ósseo. 0 membro afetado foi envolvido por uma malha ortopédica $(8 \mathrm{~cm} \mathrm{x}$ $15 \mathrm{~m}$ ) desde o casco até a porção média do osso adjacente à articulação do osso fraturado. Após, aplicou-se atadura de algodão ortopédico (15cm x 1m) sobre toda área da malha tubular seguida por atadura de gaze (10 ou $25 \mathrm{~cm}$ x 1,80m). Então, realizou-se a compressão da bandagem com esparadrapo $(10 \mathrm{~cm}$ x 4,5m), para manutenção do alinhamento ósseo. Em seguida, foram utilizados dois segmentos de canos de PVC com as pontas arredondadas e diâmetro aproximado da imobilização realizada, nas faces lateral e medial do membro, acoplados com o uso de esparadrapo seguido de nova compressão. Finalmente, foram aplicados de dois a quatro rolos de ataduras gessadas (Atadura gessada - Cremer S.A., Blumenau, SC) $(15 \mathrm{~cm} \times 3 \mathrm{~m})$ por todo o membro, com a secagem do gesso realizada com secador de cabelos. Em quatro ruminantes com fraturas proximal (Casos 14, 18 e 21) ou exposta (Caso 19) (Quadro 1), o tratamento conservativo foi a conduta terapêutica utilizada devido a impossibilidade do tratamento cirúrgico. Após os procedimentos, os ruminantes receberam fenilbutazona (Butafenil - Laboratório Calier Saúde Animal Ltda., Barcelona, Espanha) (5mg. $\mathrm{kg}^{-1}$, IV, s.i.d., 3 dias) e foram submetidos a restrição de movimentos durante todo o período de convalescência.

Em quatro animais (Casos 14, 16, 19 e 20), devido ao baixo peso corporal dos pacientes, optou-se pela utilização de abaixadores de madeira para língua (Abaixadores de madeira para língua - Theoto S.A. Indústria e Comércio, SP) (1x14cm) e esparadrapo como método de imobilização, substituindo os canos de PVC e atadura gessada. Em pequenos ruminantes adultos, com exceção do ovino com fratura de falange medial (Caso 12), e nos bovinos jovens utilizou-se a imobilização com gesso associada à tala de Thomas seguindo a metodologia de Gangl et al. (2006) com modificações. Para isso, o membro fraturado foi medido e uma barra de aço (1 cm de diâmetro) foi moldada de acordo com a anatomia do membro, deixando-se folga de $3 \mathrm{~cm}$. 0 anel de sustentação medial foi confeccionado com mangueira de gás e fixado à tala com uso de esparadrapo, que também foi utilizado para revestir todo o aço da tala. Para os membros pélvicos, o suporte da tala foi dobrado aproximadamente $30^{\circ}$ para fora do plano do anel de sustentação medial, com intuito de melhor acomodação na tuberosidade coxal; enquanto nos membros torácicos, deixaram-se as hastes de suporte retas. A fixação da tala ao membro foi feita com o uso de esparadrapo (Fig.1A e 1C). As imobilizações foram retiradas após 35 a 50 dias.

\section{Fixação externa}

Nos demais casos de fraturas proximais (acima da articulação carpo-radial ou tarso-tibial; Casos 6, 9 e 11), fratura exposta de metatarso (Caso 7) ou metacarpo (Caso 22), os ruminantes foram submetidos a protocolos anestésicos específicos para posterior uso de fixador esquelético externo (FEE) ou pinos intramedulares com hemicerclagem (PIM).

No Caso 6 optou-se pela tranquilização com cloridrato de xilazina $\left(0,05 \mathrm{mg} \cdot \mathrm{kg}^{-1}\right)$ seguida de bloqueio segmentar subaracnóide com cloridrato de ropivacaína (Cloridrato de ropivacaína 1\% - Eurofarma, São Paulo, SP) (1,5mg.kg-1). A intervenção cirúrgica consistiu na fixação dos fragmentos ósseos com um pino intramedular de $4 \mathrm{~mm}$, dois pinos de Schanz transcorticais e barras de conexão externas. No Caso 7, a medicação pré-anestésica (MPA) consistiu da associação de acepromazina (Apromazin - Syntec do Brasil Ltda, Cotia, SP) $\left(0,05 \mathrm{mg} \cdot \mathrm{kg}^{-1}\right)$ e cloridrato de xilazina $\left(0,1 \mathrm{mg}^{\mathrm{kg}}{ }^{-1}\right)$; e manutenção com cetamina (Cetamin - Syntec do Brasil Ltda., Cotia, SP) $\left(2 \mathrm{mg} \cdot \mathrm{kg}^{-1}\right)$. Devido à ocorrência de fratura transversa exposta minimamente fragmentada, a utilização de pino intramedular de Steinmann de 3,5mm e hemicerclagens com fio de aço de $0,8 \mathrm{~mm}$ foi a conduta instituída. Os demais ruminantes (Casos 9, 11 e 22) foram submetidos à mesma MPA supracitada associada à indução 
Quadro 1. Dados epidemiológicos, local da fratura, tipo de tratamento e desfecho em 22 ruminantes com fraturas em membros.

\begin{tabular}{|c|c|c|c|c|c|c|c|c|c|}
\hline Caso & Espécie & Raça & Idade & Sexo & $\begin{array}{l}\text { Peso } \\
(\mathrm{kg})\end{array}$ & Local da fratura & $\begin{array}{l}\text { Evolução clínica } \\
\text { (dias) }\end{array}$ & Tratamento & Desfecho \\
\hline 1 & Bovina & Mestiça & 2 meses & M & 34,3 & Metatarso direito & 1 & $\mathrm{GT}^{\mathrm{a}}$ & Recuperação \\
\hline 2 & Bovina & Mestiça & 5 meses & $\mathrm{F}$ & 55,6 & Metatarso direito & 3 & GT & Recuperação \\
\hline 3 & Bovina & Mestiça & 19 dias & $\mathrm{F}$ & 23,5 & Metatarso direito & 1 & GT & Recuperação \\
\hline 4 & Bovina & Gir & 8 meses & $\mathrm{F}$ & 113 & Metacarpo esquerdo & 2 & GT & Recuperação \\
\hline 5 & Ovina & Santa Inês & $>4$ anos & M & 44,7 & Metacarpo direito & 2 & GT & Recuperação \\
\hline 6 & Caprina & Marota & $>4$ anos & $\mathrm{F}$ & 16,6 & Fêmur direito & $\mathrm{NI}^{\mathrm{b}}$ & $\mathrm{FEE}^{\mathrm{c}}$ & Recuperação \\
\hline 7 & Ovina & Mestiça & 2 meses & M & 7 & Metatarso esquerdo* & NI & $\mathrm{PIM}^{\mathrm{d}}$ & Recuperação \\
\hline 8 & Caprina & Saanen & $>4$ anos & $\mathrm{F}$ & 29,3 & Metatarso esquerdo & 6 & GT & Recuperação \\
\hline 9 & Caprina & Mestiça & 6 meses & $\mathrm{F}$ & 12,5 & Tíbia direita & 1 & FEE & Recuperação \\
\hline 10 & Caprina & Mestiça & $>4$ anos & $\mathrm{F}$ & 26,8 & Metatarso direito & 6 & GT & Recuperação \\
\hline 11 & Caprina & Mestiça & 5 meses & M & 5,9 & Tíbia esquerda & 1 & FEE & Recuperação \\
\hline 12 & Ovina & Dorper & 3 anos & M & 74 & Falange medial & 20 & Gesso & Recuperação \\
\hline 13 & Caprina & Mestiça & 2 meses & M & 9,2 & Metatarso esquerdo & 1 & Tala & Recuperação \\
\hline 14 & Ovina & Mestiça & 2 meses & $\mathrm{F}$ & 5,3 & Úmero direito & 5 & Tala & Recuperação \\
\hline 15 & Bovina & Mestiça & 8 meses & $\mathrm{F}$ & 80 & Metatarso esquerdo & 1 & GT & Recuperação \\
\hline 16 & Caprina & Saanen & 2 dias & $\mathrm{F}$ & 1,1 & Tíbia esquerda & 1 & Tala & Recuperação \\
\hline 17 & Ovina & Morada Nova & 2 meses & $\mathrm{F}$ & 7,2 & Metacarpo direito & 1 & Tala & Recuperação \\
\hline 18 & Caprina & Mestiça & 5 anos & $\mathrm{F}$ & 35 & Tíbia esquerda & 4 & GT & Recuperação \\
\hline 19 & Ovina & Morada Nova & 3 dias & M & 1,2 & Rádio e ulna esquerdos* & 2 & Tala & Recuperação \\
\hline 20 & Ovina & Santa Inês & 5 dias & $\mathrm{F}$ & 3 & Tíbia esquerda & 3 & Tala & Recuperação \\
\hline 21 & Ovina & Santa Inês & 6 meses & M & 14 & Fêmur direito & 1 & GT & Abate \\
\hline 22 & Bovina & Nelore & 1 mês & M & 50 & Metacarpo direito* & 3 & FEE & Recuperação \\
\hline
\end{tabular}

a Imobilização com gesso e tala de Thomas, ${ }^{\mathrm{b}}$ não informado, ${ }^{\mathrm{c}}$ Fixador esquelético externo, ${ }^{\mathrm{d}}$ Pino intramedular com hemicerclagem. *Fratura exposta. $\mathrm{M}=$ macho, $\mathrm{F}=$ fêmea.

com propofol (Propovan - Cristália Produtos Químicos e Farmacêuticos Ltda., Itatira, SP) (4mg.kg-1) com posterior intubação e manutenção anestésica por 1,5 CAM de isoflurano (fluxo de $20 \mathrm{~mL}$. kg- de oxigênio a 100\%), em circuito semi-fechado com adsorvedor de $\mathrm{CO}_{2}$. Os procedimentos cirúrgicos consistiram na utilização de fixação esquelética externa com uso de quatro pinos transcorticais de Steinmann de 2,5mm, unidos por duas barras de polimetilmetacrilato (Fig.1E e 1F). No pós-cirúrgico, utilizou-se tramadol (Tramadon - Cristália Produtos Químicos e Farmacêuticos Ltda., Itatira, SP) (2mg.kg-1 ${ }^{-1}$ IM, q.i.d., 2 dias), cetoprofeno (Ketofen - Merial Saúde Animal Ltda., Campinas, SP) (2mg.kg-1 ${ }^{-1}$ IM; s.i.d., 5 dias) e ceftiofur (Bioxell - Vallée, Uberlândia, MG) (2,2mg.kg-1, IM, s.i.d., 5 dias). Esta terapia medicamentosa foi utilizada, também, no ovino com fratura exposta, e submetido ao tratamento conservativo (Caso 19). As imobilizações foram retiradas após 50 a 80 dias.

\section{RESULTADOS E DISCUSSÃO}

Os principais dados epidemiológicos, local da fratura, tipo de tratamento e desfecho são apresentados no Quadro 1. Todos os proprietários relataram como queixa principal a claudicação aguda, em que o animal não apoia o peso no membro afetado. Ao exame clínico havia instabilidade, edema e crepitação à palpação do local da fratura e doze ruminantes apresentaram, também, taquicardia. Em animais com fraturas expostas (Casos 7, 19 e 22), foi notória a presença de fraturas oblíquas completas e sujidades no local da perfuração da pele. No ovino com fratura de falange medial (Caso 12), o diagnóstico definitivo foi obtido por meio de radiografia, já que o único sinal clínico perceptível foi a severa claudicação de apoio.

A maior casuística ocorreu em caprinos e ovinos (16 casos; $69,5 \%$ ), e é, provavelmente, decorrente da grande população destas espécies no estado do Rio Grande do Norte (IBGE 2012), além da indiscutível facilidade de transporte destes quando comparado aos bovinos. Acredita-se que diversos casos de fraturas em ruminantes sequer sejam examinados por médicos veterinários, e que tais animais são imediatamente encaminhados para abate por opção do proprietário, por crerem que o custo-benefício do tratamento é inviável. Entretanto, pelos resultados obtidos, afirma-se que os tratamentos conservativos descritos apresentam-se como opção de baixo custo, além da fácil aquisição dos materiais utilizados para o tratamento de fraturas em pequenos ruminantes de qualquer idade ou bovinos jovens. No Brasil, os procedimentos cirúrgicos (fixação externa ou interna) permanecem com a utilização restrita a animais de considerável valor comercial (fêmeas doadoras de embrião, animais de alta produção leiteira ou de corte e machos reprodutores) ou afetivo (ruminantes de estimação), tendo como limitação o custo da anestesia inalatória e alguns materiais utilizados durante a cirurgia (Martins et al. 2001, Nóbrega et al. 2008).

No presente estudo observou-se a maior frequência de fraturas envolvendo o metacarpo ou metatarso (12 casos; $54,5 \%$ ), seguido por fraturas de tíbia ( 5 casos; $22,7 \%$ ), fêmur ( 2 casos; 9\%), falange medial (1 caso; 4,5\%), úmero ( 1 caso; $4,5 \%$ ), rádio e ulna ( 1 caso; $4,5 \%$ ). Tais resultados confirmam que fraturas distais acometendo metacarpo ou metatarso são as mais comuns em ruminantes (Rielly et al. 2005, Anderson \& St. Jean 2008, Steiner \& Anderson, 2009, Mulon 2013); enquanto outros estudos apresentam as fraturas de tíbia como as mais frequentes em bovinos (Martens et al. 1998, Gangl et al. 2006). A falta de proteção por tecidos moles acarreta maior vulnerabilidade do metacarpo e metatarso a traumas externos; sendo tais ossos também mais propensos à ruptura provocada por força de tração excessiva (p.ex. uso de correntes obstétricas em partos 
distócicos ou pelo ato de prender o membro em algum obstáculo), possivelmente resultando em interrupção da placa de crescimento (Mulon 2013). Nos casos aqui relatados, a maioria das fraturas decorreu do manuseio inadequado pelos proprietários e tratadores ou devido a traumatismos infligidos por outros ruminantes do mesmo rebanho.

As fraturas de tíbia ocorreram em cinco ruminantes e foram tratadas conservativamente (Casos 16, 18 e 20) e
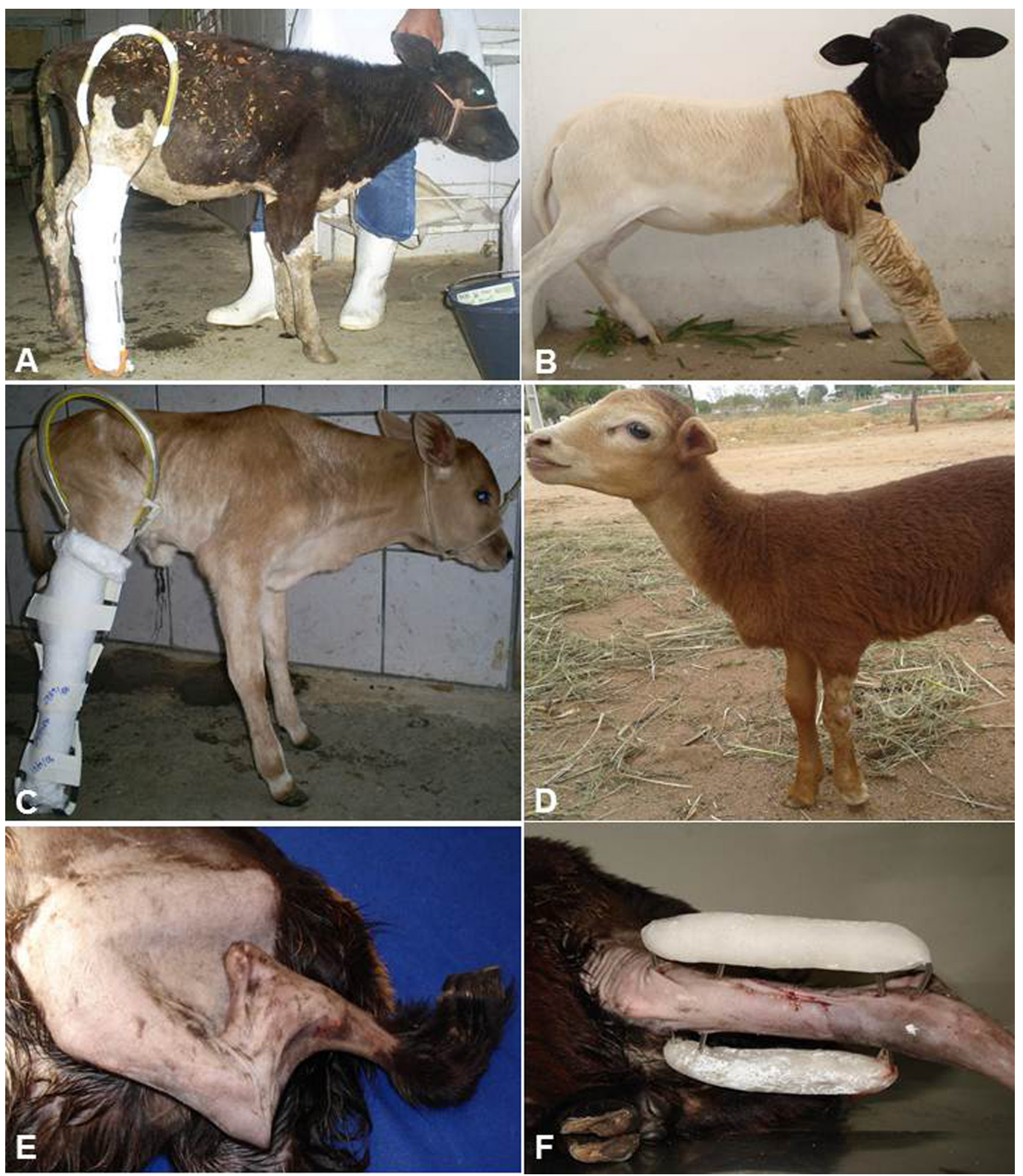

Fig.1. Fraturas em membros de ruminantes. (A) Caso 2 e (C) 3 após imobilização de fraturas de metatarso com gesso associada à tala de Thomas. (B) Ovino após 45 dias da imobilização com talas de madeira para tratamento de fratura de úmero (Caso 14). (D) Ovino com apoio nas pinças imediatamente após a retirada da imobilização (Caso 19). (E) Caprino (Caso 11) com fratura oblíqua de tíbia antes e (F) após a fixação esquelética externa. 
com o uso de FEE (Casos 9 e 11) (Fig.1E e 1F); alcançando índice de recuperação de 100\%. Em fraturas de tíbia, os tratamentos conservativos geralmente não resultam em cicatrização óssea adequada, pois não estabilizam adequadamente a articulação fêmurotíbio-patelar (Aithal et al. 2010, Mulon 2013). Entretanto, estudos anteriores em bovinos com fraturas tibiais apresentaram índice de recuperação de 42,8\% (3/7) quando eram apenas confinados em baia após a fratura (Martens et al. 1998); este índice aumentou para $45,4 \%$ (5/11) e 79,6\% (43/54) quando foi realizada a imobilização com gesso sintético e tala de Thomas (Martens et al. 1998, Gangl et al. 2006). 0 tratamento cirúrgico utilizando pinos transcorticais e fixação externa com metilmetacrilato também foi empregado com sucesso em um ovino de duas semanas de idade (Nóbrega et al. 2008) e em bovinos jovens e adultos (Martens et al. 1998, Martins et al. 2001, Mulon 2013); sendo também relatado o uso bem sucedido do fixador externo circular em bezerros (Aithal et al. 2010).

A fratura de fêmur ocorreu em dois casos (Casos 6 e 21) e apresenta prognóstico reservado em ruminantes de qualquer idade, sendo, muitas vezes, a única opção a eutanásia (Mulon 2013). 0 tratamento cirúrgico por meio de redução aberta da fratura associada à fixação interna é considerada a melhor opção terapêutica (Ewoldt et al. 2003, Nichols et al. 2010). A conduta cirúrgica instituída com o uso da associação de fixação interna (pino intramedular de $4 \mathrm{~mm}$ ) e externa (dois pinos de Schanz transcorticais e barras de conexão) é uma opção viável para o tratamento de fraturas de fêmur em caprinos, principalmente quando o animal for de baixo peso corporal e de alto valor zootécnico, como o do presente trabalho, pertencente a uma espécie em perigo de extinção. 0 tratamento conservativo de fraturas femorais apresenta-se inviável devido à incapacidade de estabilização óssea adequada, mesmo quando se associa a imobilização completa do membro com a tala de Thomas (Mulon 2013), assim como comprovado no Caso 21.

Casos isolados de fraturas de falange (Caso 12), úmero (Caso 14), rádio e ulna (Caso 19) foram tratados conservativamente e apresentaram resolução satisfatória. A utilização de imobilização com gesso até a articulação carpo-radial mostrou-se eficaz no tratamento de fratura de falange medial, assim como observado anteriormente (Anderson \& St. Jean 2008, Steiner \& Anderson 2009, Mulon 2013). Destaca-se o sucesso no tratamento conservativo de fraturas proximais localizadas no rádio e úmero (Fig.1B), já que esses ossos, devido à localização anatômica, apresentam desafio de estabilização similar ao fêmur (Vechiato et al. 2009, Mulon 2013). Entretanto, a imobilização obtida nestes dois casos foi suficiente para permitir adequada consolidação óssea. Fato este comprovado por Baird \& Adams (2014), que demonstram a possibilidade de uso bem sucedido de associação de imobilização com gesso sintético e talas de Thomas ou Walker, ou bandagem de Spica associada à tala sobre o ombro, no tratamento conservativo de fraturas proximais nos membros torácicos em bovinos.

Complicações associadas ao uso das imobilizações, como feridas cutâneas profundas, anquilose, sobrecarga do membro contralateral e laminite são citadas na literatura, sendo necessário o monitoramento diário destes pacientes (Gangl et al. 2006, Anderson \& St. Jean 2008, Mulon 2013). Em dois casos foram observadas tais complicações, ocorrendo afrouxamento dos tendões flexor digital superficial e profundo em uma bezerra da raça Gir (Caso 4), que necessitou de correção por uso de talas também no membro contralateral. Uma cabra da raça Saanen (Caso 8) apresentou necrose e perda do dígito medial do membro acometido devido ao acolchoamento insuficiente da região interdigital. Os demais ruminantes apresentaram discreta a relevante atrofia muscular (Fig.1D), sendo a tonicidade e vigor da musculatura recuperada gradativamente com restauração total da biomecânica funcional do membro acometido em até seis semanas.

0 índice de recuperação total alcançou 95,4\% (21/22). 0 alto índice de recuperação obtido neste estudo pode estar correlacionado ao enorme potencial osteogênico dos ruminantes (Steiner \& Anderson 2009), associado, nestes casos em particular, ao baixo peso corporal, idade e temperamento calmo, que podem ter contribuído para o sucesso do tratamento conservativo e cirúrgico. Tais fatos corroboram que as condutas para redução de fraturas em animais de produção apresentam prognósticos consideravelmente favoráveis (Anderson \& St. Jean 2008), mesmo considerando a delgada espessura das corticais ósseas constatadas em ovinos e caprinos, como fator limitante às condutas ortopédicas (Reilly et al. 2005). Salienta-se, ainda, que os ruminantes são considerados excelentes pacientes para o tratamento de lesões ortopédicas devido ao seu comportamento e são mais resistentes com relação a fraturas e laminite no membro contralateral, quando comparados aos equinos (Anderson \& St. Jean 2008, Steiner \& Anderson 2009, Mulon 2013).

A imobilização com gesso associada à tala de Thomas, ou apenas com talas de madeira em animais jovens, foi eficiente na redução de fraturas de metacarpo, metatarso, tíbia, úmero e rádio nos ruminantes tratados. Enquanto no caso de fratura de falange medial, a imobilização com uso apenas de gesso foi suficiente para proporcionar reparação óssea adequada. A fixação esquelética externa ou interna deve ser considerada uma opção no tratamento de fraturas metacárpicas, metatársicas, tibiais e femorais em ruminantes, principalmente quando o animal for de baixo peso corporal.

\section{REFERÊNCIAS}

Aithal H.P., Kinjavdekar P., Pawde A.M. Singh G.R. \& Setia H.C. 2010. Management of tibial fractures using a circular external fixator in two calves. Vet. Surg. 39(5):621-626.

Anderson D.E. \& St. Jean G. 2008. Management of fractures in field settings. Vet. Clin. North Am., Food Anim. Pract. 24(3):567-582.

Baird A.N. \& Adams S.B. 2014. Use of the Thomas splint and cast combination, Walker splint, and Spica bandage with an over the shoulder splint for the treatment of fractures of the upper limbs in cattle. Vet. Clin. North Am., Food Anim. Pract. 30(1):77-90.

Ewoldt J.M.I., Hull B.L. \& Ayars A.H. 2003. Repair of femoral capital physeal fractures in 12 cattle. Vet. Surg. 32(1):30-36.

Gangl M., Grulke S., Serteyn D. \& Touati K. 2006. Retrospective study of 99 cases of bone fractures in cattle treated by external coaptation or confinement. Vet. Rec. 158(8):264-268.

IBGE 2012. Instituto Brasileiro de Geografia e Estatística. Capturado em 9 abr. 2014. Online. Disponível em <http://www.ibge.gov.br/estadosat/ temas.php?sigla=rn\&tema=pecuaria2012> 
Martens A., Steenhaut M., Gasthuys F., De Cupere C., De Moor A. \& Verschootenl F. 1998. Conservative and surgical treatment of tibial fractures in cattle. Vet. Rec. 143(1):12-16.

Martins E.A.N., Galera P.D., Ribas J.A.S. \& Silveira D. 2001. Gesso sintético e pinos transcorticais na redução de fratura de tíbia em uma bezerra. Ciência Rural 31(1):145-148.

Mulon P.Y. 2013. Management of long bone fractures in cattle. In Practice 35(5):265-271.

Nichols S., Anderson D.E., Miesner M.D. \& Newman K.D. 2010. Femoral diaphysis fractures in cattle: 26 cases (1994-2005). Aust. Vet. J. 88(1/2):39-44.

Nóbrega F.S., Gianotti G.C., Alievi M.M., Beck C.A.C., Ferreira M.P., Stedile R., Dal-Bó I.S., Gonzalez P.C. \& Voll J. 2008. Osteossíntese de tíbia com fixador esquelético externo em um cordeiro. Acta Sci. Vet. 36(1):55-58.
Reilly L.K., Baird A.N. \& Pugh D.G. 2005. Enfermidades do sistema musculoesquelético, p.252-286. In: Pugh D.G. (Ed.), Clínica de Ovinos e Caprinos. Roca, São Paulo.

Spadeto Júnior O., Faleiros R.R., Alves G.E.S., Casas E.B.L., Rodrigues L.B., Loiacono B.Z. \& Cassou F. 2010. Falhas na utilização de poliacetal e poliamida em forma de haste intramedular bloqueada para imobilização de fratura femural induzida em bovinos jovens. Ciência Rural 40(4):907-912.

Steiner A. \& Anderson D.E. 2009. Fracture management in cattle, p.253258. In: Anderson D.E. \& Rings D.M. (Eds), Current Veterinary Therapy: food animal practice. Vol.5. W.B. Saunders, Philadelphia.

Vechiato T.A.F,, Siqueira R.F., Coutinho A., Marchioni G.G., Kolber M., Peixoto Jr K.C., Toffoli P.Z. \& Zanco N.A. 2009. Utilização de fixação externa em fratura de úmero em caprino. Arq. Bras. Med. Vet. Zootec. 61(5):1242-1245. 